J. Clin. Chem. Clin. Biochem.

Vol. 23, 1985, pp. $759-763$

\title{
Reaktion von direktem und indirektem Bilirubin mit Fast Red TR und verwandten Diazoniumsalzen
}

\author{
Von G. E. Hoffmann, Renate Hiefinger \\ Institut für Klinische Chemie am Städtischen Krankenhaus München-Bogenhausen, München und \\ L. Weiss \\ Institut für Klinische Chemie am Städtischen Krankenhaus München-Harlaching, München
}

(Eingegangen am 30. Mai/23. Juli 1985)

Zusammenfassung: In der vorliegenden Arbeit wird die Reaktivität von 15 verschiedenen Diazoniumsalzen gegenüber Bilirubin im Serum untersucht. Es wird gezeigt, daß die Selektivität der Bestimmung von direktem und gesamtem Bilirubin vor allem von zwei Bedingungen abhängig ist: vom pH-Wert der Reaktionslösung und von der Art und Stellung der Substituenten am Benzolring. Durch Einführung „elektronenschiebender“ Gruppen wie z. B. der $o$-Methylgruppe im Fast Red TR läßt sich die Reaktivität des Diazoniumkations gegenüber Bilirubin gezielt so verändern, daß bei pH-Werten zwischen 2 und 5 die Mitreaktion der indirekten Fraktion auf ein Minimum reduziert ist. Nach Neutralisierung auf $\mathrm{pH} 7$ läßt sich das gesamte Bilirubin bestimmen.

\section{Reaction of non-conjugated and conjugated bilirubin with Fast Red TR and related diazonium salts}

Summary: In the present study, the reaction of 15 different diazonium salts with serum bilirubin is examined. It is shown that the selectivity of the determination of non-conjugated and total bilirubin greatly depends on two conditions: the $\mathrm{pH}$ =value of the reaction mixture and the nature and position of substituents within the benzene ring. Introduction of "electron-pushing" groups such as the $o$-methyl group of Fast Red TR will change the reactivity of the diazonium cation towards bilirubin in such a way that the co-reaction of the conjugated fraction becomes negligible at $\mathrm{pH}$-values between 2 and 5 . After neutralization to $\mathrm{pH} 7$ the total bilirubin can be determined.

\section{Einfưuhrung}

Die differenzierte Bestimmung des direkten und gesamten Bilirubins im Serum erfolgt seit über $50 \mathrm{Jah}$ ren bevorzugt nach der Methode von Jendrassik \& Grof (1), bei der frisch diazotierte Sulfanilsäure als Kupplungsreagenz und Coffein als sog. „Akzelerator" verwendet werden. Geringe Reagenzienstabilität und relativ umständliche Testdurchführung haben zur Entwicklung neuerer Verfahren geführt, die vor allem darauf abzielten, die Sulfanilsäure durch stabile Diazoniumsalze und das Coffein durch Detergenzien zu ersetzen. Probleme ergaben sich hier jedoch bei der selektiven Erfassung des sog. direkten Bilirubins, da meist eine Mitreaktion des indirekten Anteils zu beobachten war (2, 3). Die Ursachen dieser mangelnden Spezifität sind bis jetzt ungeklärt.

Wir fanden vor kurzem, daß Fast Red TR unter bestimmten Bedingungen eine selektive Azokupplungsreaktion mit direktem Bilirubin eingeht (4). Dieser Effekt wurde in der vorliegenden Arbeit unter Einbeziehung weiterer Diazoniumsalze näher untersucht. 


\section{Material und Methoden}

Alle Untersuchungen wurden 1 bis $5 \mathrm{~h}$ nach Entnahme an kühl und lichtgeschützt aufbewahrten Seren von Erwachsenen und Neugeborenen durchgeführt.

Diazoniumsalze der Tetrafluorborsäure und des Zinkchlorids stammten von Sigma. St. Louis (USA), Boehringer, Mannheim und Serva, Hcidelberg, die Puffersubstanzen von Merck, Darmstadt. Dic Reagenzien für die Bestimmung von direktem und gesamtem Bilirubin nach Jendrassik \& Grof (1) wurden von Gödecke, Freiburg bezogen.

Zur Herstellung der benötigten Puffer wurde Citronensäure $(0,2 \mathrm{~mol} / \mathrm{l}) \mathrm{mit}$ Tris-hydroxymethyl-aminomethan $(0,2 \mathrm{~mol} / \mathrm{l})$ auf $\mathrm{pH}$-Wertc zwischen 2 und 7 cingestellt. Die gebrauchsfertigen Diazoniumsalze (Tab. 1) wurden darin in einer Konzentration von $5 \mathrm{mmol} / \mathrm{l}$ gelöst. In den Test wurden $0,5 \mathrm{ml}$ Reaktionsgemisch und $0,02 \mathrm{ml}$ Scrum eingesctzt.

Tab. 1. Chemische Struktur von 15 getesteten Diazonium-Verbindungen. Zur Vereinhcitlichung der Nomenklatur wird dic Position der Diazoniumgruppierung stets mit 1 bezcichnet.
Derivate des

Diazobenzols (DB)

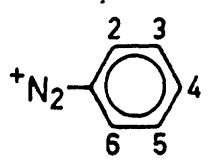

2-Chlor-DB

2-Brom-DB

2,5-Dichlor-DB

3,4-Dichlor-DB

4-Carboxy-DB

(Fast Red B)

2-Mcthyl-4-chlor-DB

(Fast Red TR)

2-Methoxy-5-chlor-DB

(Fast Red RC)

2-Methoxy-5-sulfonyl-DB

(Fast Red ITR)
4-Sulfonyl-DB (Jendrassik)

2-Mcthoxy-4-nitro-DB
Derivate des

4-Benzamido-diazobenzols (BDB)

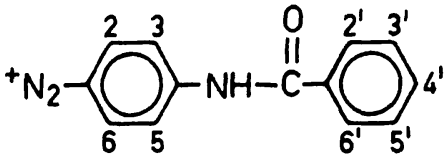

2-Mcthoxy-5-methyl-BDB

(Fast Violet B)

2,5-Diethoxy-BDB

(Fast Blue BB)

2,5-Dimethoxy-BDB

(Fast Blue RR)

Derivate des

Diazo-Azobenzols (DAB)

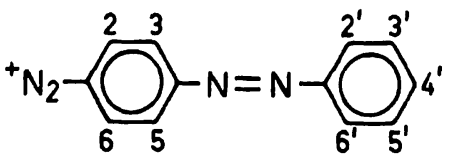

2-Methyl-2'-methyl-DAB (Fast Garnet GBC)

2-Methoxy-5-methyl-2'methyl-4'-nitro-DAB (Fast Corinth V)

\section{Ergebnisse}

In Abbildung 1 sind beispielhaft Absorptionsspektren von 4 verschiedenen Diazoniumsalzen vor und nach der Reaktion mit Bilirubin im Serum dargestellt. Mit Ausnahme der Azobenzolderivate Fast Garnet GBC und Fast Corinth $\mathrm{V}$ wiesen die getesteten Verbindungen im Spektralbereich oberhalb von $500 \mathrm{~nm}$ Wellenlänge praktisch keinc Eigenabsorption auf. Nach $\mathrm{Zu}$ -

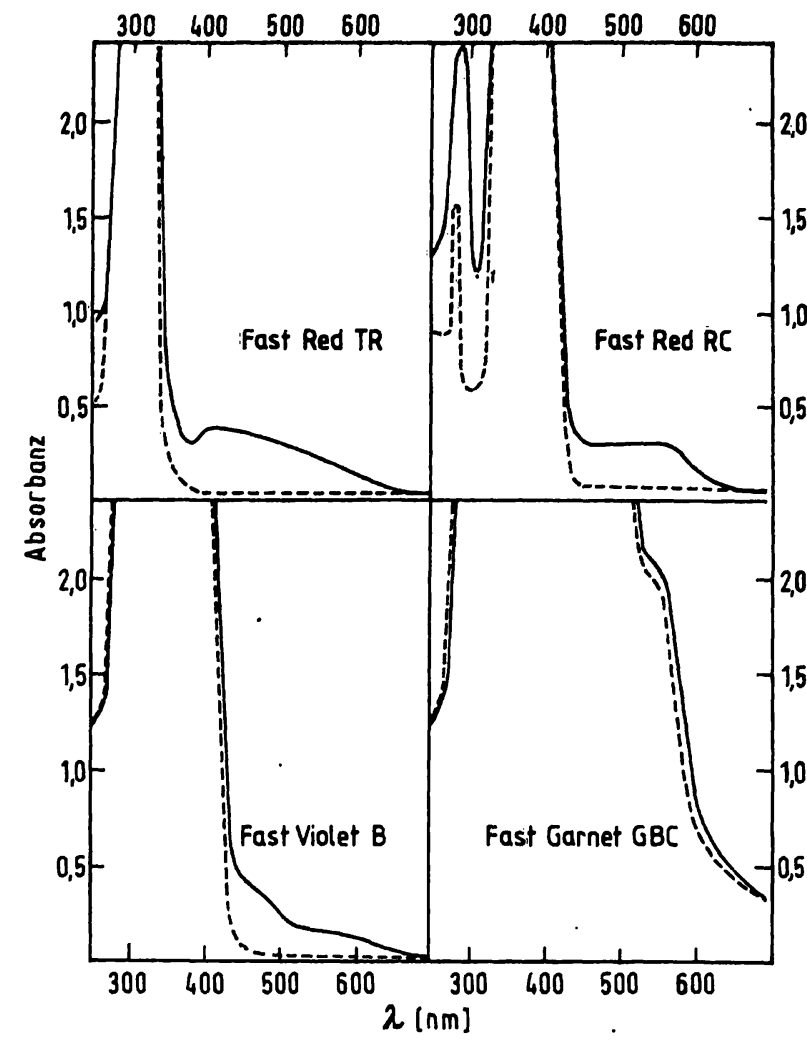

Abb. 1. Absorptionsspektren von 4 verschiedenen Diazoniumsalzen bei pH 5.2. Die unterbrochene Linie gibt das Spektrum vor, dic durchgezogene das Spektrum 10 min nach dem Start der Azokupplungsreaktion wieder. Die jeweiligen Absorptionsmaxima der Diazoniumsalże liegen oberhalb des linearen Meßbereichs.

gabe eines ikterischen Serums setzte eine unterschiedlich rasche Azokupplungsreaktion ein, die zu einer charakteristischen Zunahme der Absorbanz im Spektralbereich zwischen 500 und $650 \mathrm{~nm}$ führte.

Die Reaktionskinetik der Azokupplung war stark von Art und Stellung der Substituenten am Benzolring, vom $\mathrm{pH}$-Wert des Reaktionsgemisches und vom Anteil an direktem Bilirubin abhängig. In Abbildung 2 und 3 wird die Reaktivität von Fast Red TR und diazotiertem Dichloranilin am Beispiel einiger typischer Reaktionskinetiken verglichen.

\section{Reaktion vor der Serumzugabe}

Die Eigenabsorption von frisch gelöstem Fast Red TR blieb bei allen getesteten pH-Werten zwischen 2 und 7 konstant, während sich Diazo-Dichloranilin mit steigendem $\mathrm{pH}$-Wert zunehmend rascher verfärbte und unter Freisetzung von gasförmigem Stickstoff ein unlösliches Azokupplungsprodukt bildete (siehe Diskussion). 

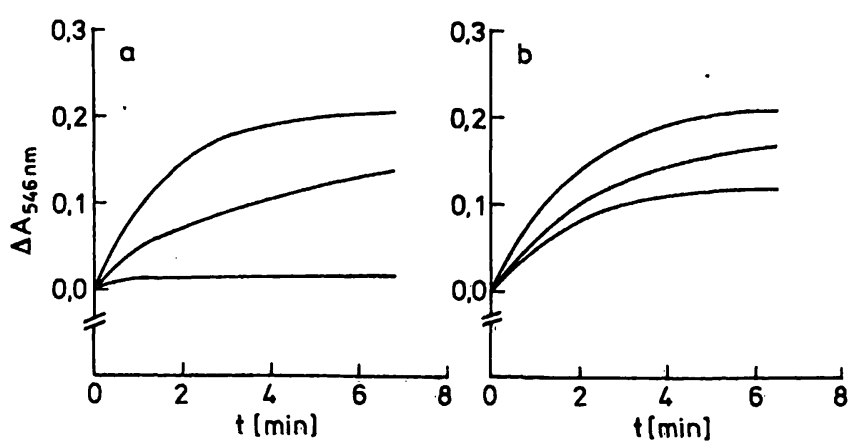

Abb. 2. Reaktion von Bilirubin mit Fast Red TR bei pH 5, 6 und 7 (untere, mittlere bzw. obere Kurve).

Serum A: Bilirubin $176 \mu \mathrm{mol} / \mathrm{l}$, direkter Anteil $2 \mu \mathrm{mol} / 1$. Ikterus Neonatorum.

Serum B: Bilirubin $180 \mu \mathrm{mol} / \mathrm{l}$, direkter Anteil 111 $\mu \mathrm{mol} / \mathrm{l}$. Hepatischer Ikterus.

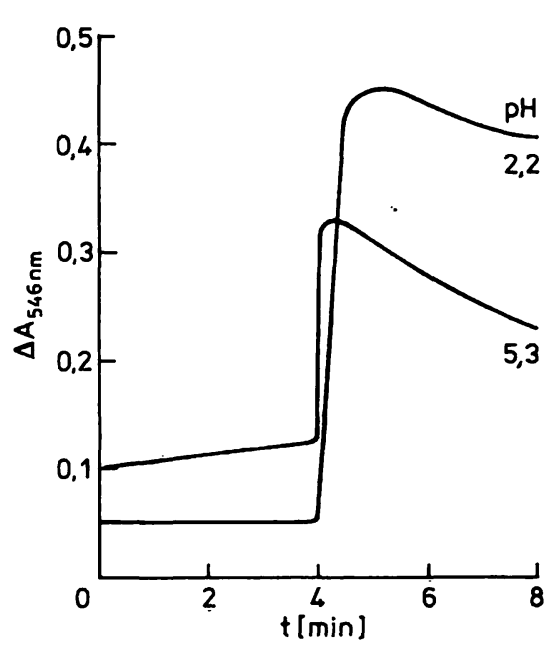

Abb. 3. Reaktion von Bilirubin mit diazotiertem 2,5-Dichloranilin bei $\mathrm{pH} 2,2$ und 5,3.

(Bilirubin gesamt $308 \mu \mathrm{mol} / \mathrm{l}$, direkter Anteil 223 $\mu \mathrm{mol} / \mathrm{l})$. Mit Neugeborenenserum ohne direktes Bilirubin verläuft die Reaktion ähnlich (nicht dargestellt).

\section{Initiale Reaktionsphase}

Nach Zugabe eines ikterischen Serums reagierte Diazo-dichloranilin mit direktem und indirektem Bilirubin bei allen getesteten pH-Werten sehr rasch, d. h. der initiale Anstieg der Absorbanz verlief äußerst steil (Abb. 3). Demgegenüber reagierte Fast Red TR grundsätzlich langsamer $a b$ und erfaßte indirektes Bilirubin bei pH-Werten unter 5,5 offenbar überhaupt nicht (Abb. 2). Zur Verdeutlichung ist in Abbildung 2 a die Reaktion von Fast Red TR mit einem Neugeborenenserum dargestellt, das praktisch nur indirektes Bilirubin enthielt. Man erkennt eine erhebliche Steigerung der Reaktivität im pH-Bereich zwischen 5 und 7. Bei pH 5 beträgt die Mitreaktion von indirektem Bilirubin weniger als $10 \%$.

\section{Erreichen des Endpunkts}

Während sich bei Fast Red TR der Endpunkt der Farbreaktion nur langsam einstellte, erreichte Diazodichloranilin innerhalb von Sekunden ein Maximum. Der gebildete Bilirubin-Azofarbstoff war im ersten. Fall stundenlang stabil, während er im zweiten Fall nach wenigen Minuten wieder zerfiel und zwar umso rascher, je höher der pH-Wert lag (Abb. 3).

Auch die übrigen in Tabelle 1 aufgeführten Diazoniumsalze wurden in gleicher Weise wie Fast Red TR und Diazo-dichloranilin hinsichtlich ihrer Reaktivität und Stabilität untersucht. Tabelle 2 gibt einen Überblick über die Versuchsergebnisse. Zur besseren Übersicht wird in der Tabelle das Verhalten im stark sauren, schwach sauren und neutralen $\mathrm{pH}$-Bereich durch Symbole beschrieben. Messungen bei alkalischen $\mathrm{pH}$ Werten wurden wegen Instabilität der Reagenzien nicht durchgeführt.

Tab. 2. Azokupplung von direktem und indirektem Bilirubin im Serum bei 3 verschiedenen pH-Werten. Als Probenmaterialien wurden Seren von Neugeborenen (vorwiegend indirektes Bilirubin) und von Patienten mit hepatischem Ikterus (vorwiegend direktes Bilirubin) verwendet.

$$
\begin{aligned}
& \text { Zeichenerklärung: - = keine Reaktion } \\
& +\quad=\text { deutliche Reaktion (vgl. } \\
& \text { Abb. 2) } \\
& ++=\text { Überreaktivität } \\
& \text { Abb. 3). }
\end{aligned}
$$

\begin{tabular}{|c|c|c|c|c|c|c|}
\hline \multirow[t]{2}{*}{ Diazoniumsalze } & \multicolumn{3}{|c|}{$\begin{array}{l}\text { Direktes } \\
\text { Bilirubin }\end{array}$} & \multicolumn{3}{|c|}{$\begin{array}{l}\text { Indirektes } \\
\text { Bilirubin }\end{array}$} \\
\hline & $\mathrm{pH} 2$ & pH 5 & $\mathrm{pH} 7$ & $\mathrm{pH} 2$ & pH 5 & $\mathrm{pH} 7$ \\
\hline $\begin{array}{l}\text { Halogenderivate } \\
\text { des Anilins, } \\
\text { Fast Red B }\end{array}$ & + & ++ & ++ & + & ++ & ++ \\
\hline $\begin{array}{l}\text { Diazo-Sulfanilsäure } \\
\text { Diazo-p-amino- } \\
\text { benzoesäure } \\
\text { Fast Red RC und ITR }\end{array}$ & + & + & + & - & + & + \\
\hline $\begin{array}{l}\text { Fast Red TR } \\
\text { Fast Violet B }\end{array}$ & + & + & + & - & - & + \\
\hline
\end{tabular}

Die Auswertung der Experimente ergab, daß Reaktivität und Instabilität stets in engem Zusammenhang standen und mit steigendem $\mathrm{pH}-$ Wert zunahmen. Halogenierte Diazoniumsalze besaßen die höchste, methylierte Verbindungen die geringste Reaktivität. Das Diazoniumsalz der Jendrassik-Grof-Reaktion lag etwa in der Mitte. 
tionsbedingungen finden, unter denen nur direktes Bilirubin erfaßt wurde. Diazotierte Sulfanilsäure und p-Aminobenzoesäure wiesen eine derartige Selektivität bei pH-Werten unter 3 auf, Fast Red RC und ITR bei pH-Werten unter 4,5, Fast Red TR und Fast Violet $\mathrm{B}$ bei $\mathrm{pH}-$ Werten unter 5,5.

Als ungeeignet für die Bilirubinbestimmung erwiesen sich Fast Blue BB und RR wegen schlechter Löslichkeit sowie Fast Corinth V und Fast Garnet GBC wegen ihrer hohen Eigenabsorption bei $500-600 \mathrm{~nm}$ Wellenlänge.

\section{Diskussion}

Das Gesamtbilirubin des Serums setzt sich aus 4 verschiedenen Fraktionen zusammen: dem unkonjugierten $\alpha$-Bilirubin, das über hydrophobe Wechselwirkungen an Albumin gebunden ist, dem mono- und diglucuronidierten $\beta$ - bzw. $\gamma$-Bilirubin, das in gelöster Form vorliegt sowie einer kovalent an Albumin gebundenen $\delta$-Fraktion $(5,6)$. Die klassische Bilirubinbestimmungsmethode von Jendrassik \& Grof (1) unterscheidet allerdings nur 2 Fraktionen, das hydrophilere „direkte“ Bilirubin $(\beta, \gamma, \delta)$, das im wäßrigen Milieu rasch reagiert und das hydrophobere ,indirekte" Bilirubin, das üblicherweise erst durch Zusatz von Lösungsvermittlern erfaßt wird $(1,7)$.

Die vorliegende Arbeit bestätigt unsere frühere Beobachtung (4), nach der das gebrauchsfertige Diazoniumsalz Fast Red TR unter bestimmten Bedingungen selektiv mit direktem Bilirubin reagierte.

Neben Fast Red TR waren auch Fast Violet B, Fast Red RC und ITR sowie diazotierte $p$-Aminobenzoesäure für die selektive Erfassung von direktem und indirektem Bilirubin geeignet. Gegenüber der diazotierten Sulfanilsäure besaßen Fast Red TR und Fast Violet B sogar den Vorteil, daß sie nicht nur im stark sauren $\mathrm{pH}$-Bereich sondern bis herauf zu einem $\mathrm{pH}$ Wert von etwa 5,5 eine Selektivität für direktes Bilirubin aufwiesen. Unter diesen milden Bedingungen konnten störende Präzipitationen von Serumproteinen ohne Zusatz von Lösungsvermittlern vermieden werden.

Ein Vergleich der getesteten Diazoniumsalze zeigte, daß „elektronenziehende“ Gruppen (z. B. Halogene) die Reaktivität steigerten, während „elektronenschiebende" Substituenten (z. B. Methylgruppen) reaktionsverlangsamend wirkten. Es liegt deshalb nahe, anzunehmen, daß die Reaktivität und damit Selektivität der Verbindungen durch die Elektronendichte am Diazoniumkation wesentlich beeinflußt wurde. Bei erhöhter Elektronendichte war der elektrophile An- griff am Bilirubin erschwert (Beispiel Fast Red TR und Fast Violet B), so da $B$ indirektes Bilirubin schlecht umgesetzt wurde. Bei geringerer Elektronendichte dagegen war die Reaktivität des Diazoniumkations so hoch (Beispiel Diazo-dichloranilin, Fast Red B), daß die Selektivität verrlorenging. In diesen Fällen kam es zu der in Abbildung 3 dargestellten „Überreaktivität". Folgende Nebenreaktionen wurden hierbei beobachtet (8):

1. Reagenzienverfall: Abspaltung von gasförmigem Stickstoff aus der gelösten Diazoniumverbindung durch sog. „Phenolverkochung " und anschließende Kupplungsreaktion des Diazoniumsalzes mit dem entsprechenden Phenol (Bildung eines schwerlöslichen Azofarbstoffs).

$\mathrm{Ar}-\mathrm{N}_{2}^{+}+\mathrm{H}_{2} \mathrm{O} \rightarrow \mathrm{ArOH}+\mathrm{N}_{2}+\mathrm{H}^{+}$

$\mathrm{Ar}-\mathrm{N}_{2}^{+}+\mathrm{ArOH} \rightarrow \mathrm{Ar}-\mathrm{N}_{2}-\mathrm{Ar}-\mathrm{OH}+\mathrm{H}^{+}$

(Ar $=$ aromatischer Rest, $\mathrm{N}_{2}^{+}=$Diazonium-Kation $)$

2. Bildung instabiler Bilirubinfarbstoffe

Azokupplung mit dem Stickstoff im Pyrrolring des Bilirubins (Triazenbildung):

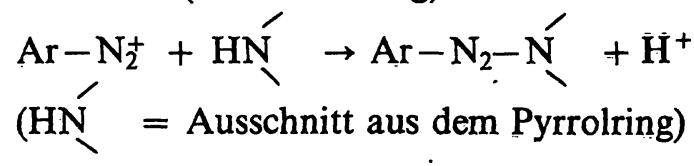

Triazene können entweder unter Stickstoffabspaltung zerfallen oder durch Umlagerung in andere Produkte mit höherer Stabilität übergehen (8).

\section{Schlußfolgerungen}

Zusammenfassend kann festgestellt werden, daß es durchaus möglich ist, direktes Bilirubin mit stabilen Diazoniumsalzen selektiv zu erfassen, wenn der $\mathrm{pH}$ Wert der Reaktionslösung und die Reaktivität des Diazoniumkations aufeinander abgestimmt sind. Für Messungen im pH-Bereich zwischen 5 und 7 ist es erforderlich, die Elektronendichte am Diazoniumkation durch Einführung „elektronenschiebender" Substituenten (Methyl-, Ethyl-, Phenylgruppen u. v. a.) so weit heraufzusetzen, daß die Mitreaktion von indirektem Bilirubin auf ein Minimum reduziert wird. Theoretisch ist hierfür eine Vielzahl von Diazoniumsalzen einsetzbar. In der Praxis hat es sich allerdings gezeigt, daß die hier getesteten 15 Verbindungen hinsichtlich Löslichkeit, Höhe des Meßsignals, Vermeidung störender Begleitreaktionen (z. B. Azokupplung mit Serumalbumin) usw. noch nicht den heutigen Anforderungen der klinischen Chemie genügen; an der Synthese eines Diazoniumsalzes, das alle diese Bedingungen in optimaler Weise erfüllt, wird zur Zeit gearbeitet. 


\section{Literatur}

1. Jendrassik, L. \& Grof, P. (1938) Biochem. Z. 297, 81-89.

2. Ostrow, J. D. \& Boonyapist, S. T. (1978) Biochem. J. 173, $263-267$.

3. Bowie, L. J., Dohnal, J. C. \& Kirkpatrick, P. B. (1982) Clin. Chem. 28, 385-386.

4. Hoffmann, G. E. \& Weiss, L. (1983) J. Clin. Chem. Clin. Biochem. 21, 31-33.

5. Weiss, J. S., Gantam, A., Lauff, J. J., Sundberg, M. W., Jathow, P., Boyer, J. L. \& Seligson, D. (1983) New England J. Med. 309, 147-150.
6. Lauff, J. J., Kasper, M. E. \& Ambrose, R. T. (1983) Clin. Chem. 29, 800-805.

7. Richterich, R. \& Colombo, J. P. (eds.) (1978) Klinische Chemie, S. Karger Verlag, Basel.

8. Fieser, L. F. \& Fieser, M. (eds.) Organische Chemie, Verlag Chemie, Weinheim (1974).

Dr. G. E. Hoffmann

Institut für Klinische Chemie am

Städtischen Krankenhaus

München-Bogenhausen

Englschalkinger Straße 77

D-8000 München 81 

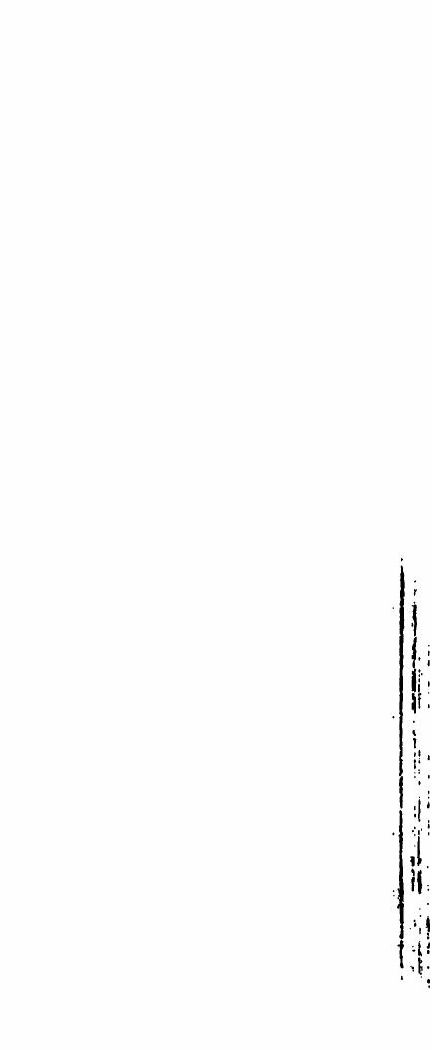